\title{
Ultra-Wide Field Angiography Improves the Detection of Proliferative Diabetic Retinopathy Progression
}

Gisung Son, Suchan Lee, Hee Young Jung, Kyung Min Lee, Young Sook Park, Joonhong Sohn and Duck Jin Hwang*

HanGil Eye Hospital, Incheon, Korea

\begin{abstract}
Objectives: To investigate patients with Proliferative Diabetic Retinopathy (PDR) using ultra-wide field fluorescein angiography (UWFA) images and to compare the detection rate of PDR progression using conventional seven standard field angiography (7SF) images.
\end{abstract}

Methods: One hundred and one eyes of 67 patients with PDR who underwent fluorescein angiography using the Optos Optomap Panoramic 200A imaging system were included. Three hundred three images of 101 eyes at different time points (initial, Post-Panretinal Photocoagulation [PRP], and follow-up) were evaluated. In comparing 101 follow-up with 101 post-PRP images, we found newly developed Neovascularization (NNV) and newly developed non-perfusion (NNP) areas, which were then analyzed using two different methodologies: UWFA and 7SF. NNVs were counted while NNP area was measured in pixels using ImageJ software.

Results: UWFA detected 21 eyes with NNV, which was approximately twice the number of eyes detected using 7SF (11 eyes). Ten eyes presented with NNV outside the 7SF area with apparently clean 7SF without NNV. UWFA detected 25 eyes with NNP areas, which was more than the six eyes detected using 7SF (19 eyes). Six eyes presented with NNP areas outside the 7SF, which were apparently fully perfused by 7 SF. In addition, UWFA detected 2.59 times more NNP area than that by 7SF.

Conclusion: Compared with conventional 7SF imaging, UWFA shows significantly improved retinal visualization and improves progression detection rates in patients with PDR.

Keywords: Neovascularization; Nonperfusion; Proliferative diabetic retinopathy; Seven standard field; Ultra wide field angiography

\section{Introduction}

Diabetic Retinopathy (DR) is the number one cause of vision loss in adults over 20 [1]. By 2050, the number of Americans with $\mathrm{DR}$ and vision threatening DR is expected to increase to 16 million and 3.4 million, respectively [2]. Current principles for treating DR depend largely on remote seminal clinical trials such as The Diabetic Retinopathy Study and Early Treatment Diabetic Retinopathy Study (ETDRS) [1]. We infer risks for progression, visual loss, and response to treatment in individual patients based on these previously established databases.

In the ETDRS, fluorescein angiography was included in the study protocol to identify sources of fluorescein leakage in macular edema and to guide the application of laser burns for macular photocoagulation treatment [3]. This study applied seven standard field (7SF) protocol in which seven circles (three horizontally across the macular and four around the optic nerve), visualizing $30^{\circ}$ of retina with each circle and $70^{\circ}$ of the retina combined, were implemented. However, this protocol has several limitations. For instance, even with today's modern digital cameras, it is cumbersome to obtain the appropriate level of patient cooperation, and good quality images, and it is technically difficult to visualize the peripheral retina [4].

Retinal imaging techniques have made a quantum leap since the ETDRS. Nowadays, we use digital imaging systems routinely instead of $35-\mathrm{mm}$ film, which has been proven agree excellently with traditional film systems [5-7]. Advancements in peripheral retina visualization are also remarkable; of note, the recently introduced Optos optomap (Optos Plc, Scotland, UK) has made Ultra-Wide Field Angiography (UWFA) possible by combining scanning laser ophthalmoscope and an ellipsoid mirror. With non-contact, non-mydriatic, panoramic fundus imaging, UWFA visualizes up to $200^{\circ}$ of the retina within a single, highresolution scan. UWFA covers almost $82 \%$ of the retina surface [8], and not only includes the entire area of the 7SF protocol used in ETDRS but also visualizes retina anterior to the equator, scanning more than twice the area of traditional 7SF [9].

UWFA has demonstrated great utility in the diagnosis and management of a range of retinal conditions [10]. A few studies have been conducted using UWFA for managing DR. These studies have detected peripheral DR pathologies and have attempted to develop meaningful associations between pathologies and macular edema and have also tried to guide targeted laser therapies [11-14]. Of note, Wessel et al. [13] proved the superior capability of UWFA for finding peripheral retinal pathologies compared to the 7SF protocol used in ETDRS. By using 218 UWFA images of 118 patients, they compared the area of retinal pathology in pixels using the two different methodologies (7SF and UWFA) by overlaying simulated 7SF images on subtracted UWFA images. Using the simulated 7SF template, they reported that UWFA detected 3.9 times more Non-Perfusion (NP) area, 1.9 times more neovascularization $(\mathrm{NV})$ area, and 3.8 times more post-panretinal photocoagulation (PRP) area than that by $7 \mathrm{SF}$

*Corresponding author: Duck Jin Hwang, MD, Department of Ophthalmology, HanGil Eye Hospital, Bupyeong-daero, Bupyeong-gu, Incheon, Gyeonggi-do, Korea Tel: +82-32-503-3322; Fax: +82-32-504-3322; E-mail: hallelu7@gmail.com

Received August 27, 2014; Accepted September 28, 2014; Published October 06, 2014

Citation: Sohn G, Lee S, Jung HY, Lee KM, Park YS, et al. (2014) Ultra-Wide Field Angiography Improves the Detection of Proliferative Diabetic Retinopathy Progression. J Diabetes Metab 5: 446 doi:10.4172/2155-6156.1000446

Copyright: (๑) 2014 Sohn G, et al. This is an open-access article distributed under the terms of the Creative Commons Attribution License, which permits unrestricted use, distribution, and reproduction in any medium, provided the original author and source are credited. 
Citation: Sohn G, Lee S, Jung HY, Lee KM, Park YS, et al. (2014) Ultra-Wide Field Angiography Improves the Detection of Proliferative Diabetic Retinopathy Progression. J Diabetes Metab 5: 446 doi:10.4172/2155-6156.1000446

Page 2 of 5

without taking conventional 7SF protocol photographs. However, their results are cross-sectional values taken at a specific instant. As DR adopts a chronic clinical course, both regular checks up and accurate retinal evaluation at each visit are critical to identify treatable retinal lesion so that timely and appropriate interventions can be initiated [15]. In this study, we determine whether DR progression is better detected using UWFA than 7SF angiography. Therefore, by using the same methodology as the aforementioned study, we performed serial follow-ups of eyes by using UWFA and compared the results of retinal pathologies to a simulated 7SF template. To our knowledge, no previous study has attempted to elucidate whether UWFA improves the detection rates of DR progression over traditional 7SF.

\section{Patient and Methods}

\section{Subjects}

In this retrospective study, we reviewed the medical records of patients who received PRP for DR at the HanGil Eye Hospital. The review period included all consecutive patients treated with PRP between January 2011 and June 2014, who met the following inclusion criteria: (1) age greater than 30 years; (2) newly diagnosed with PDR; (3) no history or clinical evidence of prior PRP; (4) no presence of vitreous hemorrhage; and (5) undergone UWFA $\geq 3$ times using the Optos Optomap Panoramic 200A imaging system (Optos Plc). Major exclusion criteria included the following: (1) other retinal diseases aside from DR; (2) previous treatment for diabetic macular edema with focal/grid laser or intravitreal injection within the past 6 months; (3) any intraocular surgery within the past 6 months or previous pars plana vitrectomy; and (4) severe cataracts or media opacity that could influence analyzing UWFA images. The following data were collected for each patient using an electronic medical record review: age, sex, duration of diabetes, insulin dependency, hemoglobin A1c, comorbid hypertension, follow-up period, and visual acuity changes (Table 1).

\section{PRP treatment}

All patients who had PDR received PRP using a pattern scanning laser (PASCAL; Opti-Media Corp., Santa Clara, CA, USA) system. All PRP procedures were performed in a dark room approximately 30 minutes after the study eye was pharmacologically dilated with $1 \%$ tropicamide and $2.5 \%$ phenylephrine. All eyes were anesthetized with topical $0.5 \%$ proparacaine eye drops. PASCAL laser photocoagulation was performed using the Super Quad 160 fundus laser lens (Volk Optical, Inc., Mentor, OH, USA) with an approximate $2 \times$ spot-size magnification. Settings were as follows: $200 \mu \mathrm{m}$ spot size, $20 \mathrm{~ms}$ pulse duration, and power increased from $300 \mathrm{~mW}$ until a gray-white lesion was attained. Burns were placed one burn width apart, and the total

\begin{tabular}{|l|c|}
\hline Characteristic $(\mathrm{n}=101)$ & \\
\hline Age, mean \pm SD (range) & $61.05 \pm 11.04(37-83)$ \\
\hline Sex, $\mathrm{n}(\%)$ & $42(62.69)$ \\
Male & $25(37.31)$ \\
\hline Female & $11.35 \pm 7.17(1-30)$ \\
\hline Diabetes & $13(19.40 \%)$ \\
Duration in years, mean \pm SD (range) & $7.78 \pm 1.40(5.8-11.0)$ \\
Insulin dependency, $\mathrm{n}(\%)$ & \\
HbA1c $(\%)$, mean \pm SD (range) & $58,(86.57 \%)$ \\
\hline $\begin{array}{l}\text { Comorbidity } \\
\text { Hypertensions, } \mathrm{n}(\%)\end{array}$ & $286.91 \pm 136.72(95-565)$ \\
\hline Follow-up period in days, mean \pm SD (range) & $-0.052 \pm 0.22$ \\
\hline Visual acuity changes, mean \pm SD (range) & \\
\hline
\end{tabular}

SD: Standard Deviation

Table 1: Baseline patient characteristics. number of spots was approximately $2400-4000$. All patients completed the entire treatment in two or three sessions.

\section{Image analysis}

Images were digitally captured using the Optos V2 Vantage Review Software and subsequently compressed into high-quality JPEG files. Images taken at three different visits were named initial, post-PRP, and follow-up images (Figure 1). Using Adobe software (Photoshop, Inc, San Jose, CA, USA), we created two simulated fields for UWFA and 7SF. For UWFA, an elliptical area was drawn on most of the angiographic field. For 7SF, seven $30^{\circ}$ circles were combined digitally as described in the ETDRS. These templates were then overlaid on the angiographic image to identify both the entire UWFA area and the potential 7SF viewable area, and the final image was then evaluated (Figure 2).

Two retinal pathologies-NV and NP-were recorded according to the methodology, 7SF and UWFA, on which it was detected. First, initial images at the time of diagnosis were analyzed in a crosssectional manner. Second, follow-up images were compared with postPRP images with respect to newly developed retinal pathologies. NV and NP found on follow-up images that were not originally observed on post-PRP images were recorded as NNV (newly-developed neovascularization) and NNP (newly-developed nonperfusion) (Figures 3 and 4). Two masked, trained graders (G. Son, and S. Lee) independently analyzed each UWFA image for two distinct retinal pathologies: (1) NV and (2) retinal NP (hypofluorescent

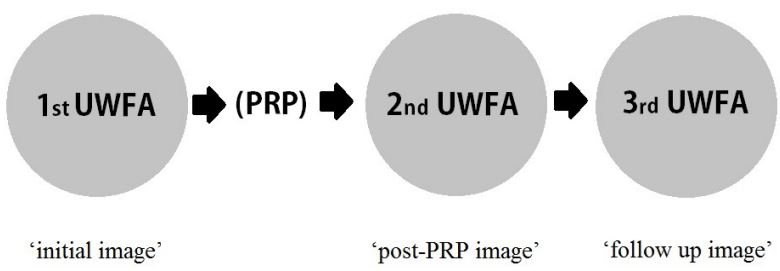

UWFA: Ultra-Wide Field Angiography; PRP: Panretinal Photocoagulation.

Figure 1: Initial images were obtained at the time of proliferative diabetic retinopathy diagnosis. Post-PRP images were obtained after the patient received panretinal photocoagulation. Follow-up images were obtained at least 3 months after post-PRP image acquisition.

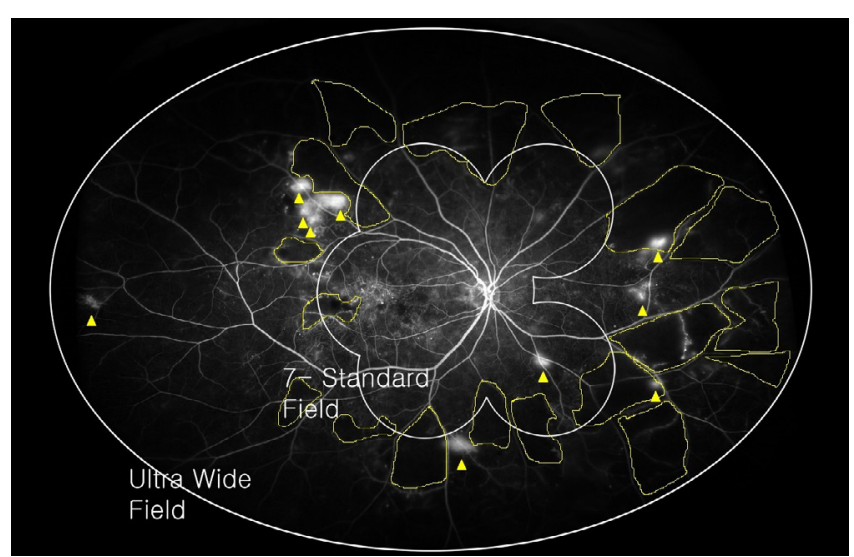

PRP: Panretinal Photocoagulation; NV: Neovascularization; NP: Non-Perfusion Area; 7SF: Seven Standard Field; UWFA: Ultra-Wide Field Angiography

Figure 2: Examples of how we set up 2 different simulated templates. Several NVs are identified with arrow head. NP area is drawn separately by hand with a yellow line. Note most of pathologies are located outside of 7SF area. 
Citation: Sohn G, Lee S, Jung HY, Lee KM, Park YS, et al. (2014) Ultra-Wide Field Angiography Improves the Detection of Proliferative Diabetic Retinopathy Progression. J Diabetes Metab 5: 446 doi:10.4172/2155-6156.1000446

Page 3 of 5

area of at least $1 / 2$ disc diameter). In each image, NVs were counted individually and NP area was measured in pixels for both the UWFA and the inlaid 7SF template using ImageJ Software (Shareware made by National Institute of Health, USA; downloaded from http://imagej. nih.gov). The data were then averaged between the two graders to obtain final values for analysis. Pearson correlation coefficients were calculated and a value greater than 0.80 was considered strong intergrader correlation.

\section{Ethics statement}

The study was approved by the institutional review board of HanGil Eye Hospital, and the study was carried out in accordance with the tenets of the Declaration of Helsinki.

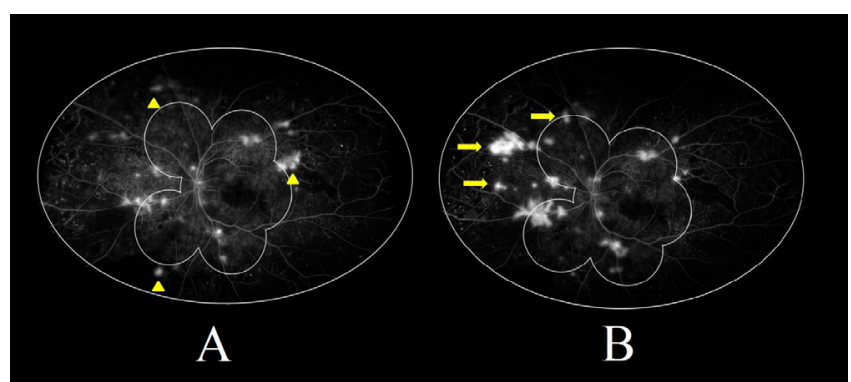

PRP: Panretinal Photocoagulation; NNV: Newly Developed Neovascularization Figure 3: Newly developed NV. A. Post-PRP image showing several neovascularization areas both within and outside the 7SF area. Note the arrowhead indicating that new vessels that regressed after the 6-month followup. B. Follow-up image showing a few changes in the distribution of new vessels. Note the arrows indicating the NNV, which were not found on the post-PRP image.



PRP: Panretinal Photocoagulation; NNP: Newly Developed Non-Perfusion

Figure 4: Newly developed NP. A. Post-PRP image showing nonperfusion areas using UWFA. Nonperfusional areas are distributed mainly throughout the midperiphery of the retina, whereas some discrete areas are located in the far periphery (arrow). B. Follow-up image showing a few NNP areas. Areas outlined in blue are NNP areas that were not found on the previous image.

\section{Statistical analyses}

Statistical analyses were performed using a commercially available software package (IBM SPSS Statistics 18; SPSS Inc., Chicago, IL, USA). Significant differences between the two different field's images were evaluated using the Pearson's Chi square test and paired t-test on initial angiography, and statistical significance was defined as $\mathrm{P}<0.05$.

\section{Results}

Among 1402 eyes in which UWFA was performed in 3.5 years in this hospital, we extracted data from 140 eyes of 70 PDR patients that serially underwent UWFA more than three times. Twenty-seven vitrectomized eyes, five eyes with medial opacity (cataract, vitrous hemorrhage), four eyes with concomitant non-diabetic retinal disease (e.g., branch retinal vein occlusion), and three eyes with artifacts (eyelashes, eyelid) were excluded. One hundred and one eyes of 67 patients were finally used in the study, and 303 image files obtained from identical eyes at three different visits were extracted and analyzed retrospectively.

$\mathrm{NV}$ and NP present on initial images were analyzed on an area basis using 7SF and UWFA (Table 2). Among 101 initial images, NV was identified in 50 eyes using 7SF, while UWFA identified 57 eyes; seven eyes had NV outside the 7SF template. Thus, 2.59 cases of NV were counted on 7SF fields, while 4.25 cases of NV were counted on UWFA, resulting in 1.66 more cases of NV identified on UWFA on average. NV counts showed great intergrader correlation coefficients, 0.91 in 7SF area and 0.88 in UWFA area each. All 101 eyes diagnosed with PDR had NP areas identified on 7SF and UWFA before treatment. NP area using 7 SF was $9.45 \times 104$ pixels on average, while NP area using UWFA was $15.37 \times 104$ pixels in average. Therefore, UWFA detected 1.62 times more NP area than that by 7SF. Area of NP in pixels also showed great intergrader correlation coefficients, 0.87 in 7SF area and 0.81 in UWFA area each.

To analyze NNV, post-PRP images and follow-up images were simultaneously displayed on screen. NNV shown on follow-up images, which did not appear on post-PRP images, were recorded and further analyzed by using both 7SF and UWFA (Table 3). Eleven eyes had NNV on 7SF, while 21 eyes had NNV on UWFA. In 10 eyes, the NV area was located outside the 7SF template (Figure 3). Among the 11 eyes with NNV found on 7SF, five eyes had NV confined within the 7SF, while six eyes had NV lesions distributed within and beyond the 7SF border. Overall, UWFA detected 2.09 times more NNV area than that by $7 \mathrm{SF}$ on average.

A similar analysis was performed for NNP. In addition to counting incidence, the area of NNP was directly measured using the ImageJ program. NNP areas were measured and analyzed by area according to

\begin{tabular}{|l|c|c|c|}
\hline & $7 S F$ area & UWFA area & $\begin{array}{c}\text { Eyes with lesion } \\
\text { outside of } 7 S F\end{array}$ \\
\hline Eyes with NV, numbers & 50 & 57 & 7 \\
\hline $\begin{array}{l}\text { NV counts in average } \\
\left(I C C^{* *}, P \text { value }\right)\end{array}$ & 2.59 & 4.25 & - \\
\hline Eyes with NP, numbers & $(0.91,<0.001)$ & $(0.88,<0.001)$ & - \\
\hline Area of NP in pixels, average $\left(I C C C^{* *}, P\right.$ value $)$ & $101 \ddagger$ & $101 \ddagger$ & 0 \\
\hline
\end{tabular}

${ }^{*}$ Ratio of UWFA area to 7SF area.

**ICC (Intergrader Correlation Coefficient): Pearson's correlation coefficient was used.

†Paired $t$-test was used to compare the average between 7SF and UWFA

$\ddagger$ All 101 eyes diagnosed with Proliferative Diabetic Retinopathy (PDR) had a non-perfusion area.

UWFA: Ultra-Wide Field Angiography; SF: Standard Field; NV: Neovascularization; NP: Non-Perfusion

Table 2: Comparison of ultra-wide field angiography with 7 standard field angiography at initial angiography. 


\begin{tabular}{|c|c|c|c|c|}
\hline & & 7SF area & UWFA area & Ratio ( $P$ value $)$ \\
\hline \multirow[b]{2}{*}{ NNV } & Eyes with NNV, number & 11 & 21 & $0.52(P<0.001)^{*}$ \\
\hline & $\begin{array}{c}\text { Average of NNV, number } \\
\left.\text { (ICC }{ }^{* \star}, P \text { value }\right)\end{array}$ & $\begin{array}{c}0.23 \\
(0.92,<0.001)\end{array}$ & $\begin{array}{c}0.48 \\
(0.88,<0.001)\end{array}$ & $\begin{aligned} 2.09(P & =0.001) \dagger \\
& -\end{aligned}$ \\
\hline \multirow[b]{2}{*}{ NNP } & Eyes with NNP, numbers & 19 & 25 & $1.32(P<0.001)^{*}$ \\
\hline & $\begin{array}{l}\text { Average of NNP, pixels } \\
\quad\left(\mathrm{ICC}^{\star *}, P \text { value }\right)\end{array}$ & $\begin{array}{c}5127.82 \\
(0.88,<0.001)\end{array}$ & $\begin{array}{c}17542.54 \\
(0.82,<0.001)\end{array}$ & $\begin{aligned} & 2.59(P=0.002) \dagger \\
&-\end{aligned}$ \\
\hline
\end{tabular}

*Pearson's Chi-square test was used to compare detection rates between 7SF and UWFA.

** ICC(Intergrader Correlation Coeffecient) : Pearson's correalation coefficient was used.

†Paired $t$-test was used to compare average values between 7SF and UWFA.

SF: Standard Field; UWFA: Ultra-Wide Field Angiography; NNV: Newly Detected Neovascularization; NNP: Newly Detected Non-Perfusion.

Table 3: Newly detected neovascularization and non-perfusion.

7SF and UWFA (Table 3). In total, 19 eyes presented with NNP on 7SF, while 25 eyes showed NNP on UWFA. In six eyes, the NNP was located exclusively outside the 7SF template (Figure 4). 7SF identified 5127.82 pixels with NNP, while UWFA identified 17542.54 pixels with NNP on average. Overall, UWFA detected 2.59 times more NNP area than that by $7 \mathrm{SF}$ on average.

\section{Discussion}

The utility of UWFA for visualizing peripheral retina analysis was proven recently in a retrospective study performed by Wessel et al. [13]. They showed wider visualization of UWFA elucidates 1.9 times greater $\mathrm{NV}$ and 3.9 times greater NP area than a simulated 7SF template. The data we analyzed using initial images showed that UWFA, averaged per eye, discloses 1.66 more NV and 1.62 times more NP area than 7SF (Table 3). This supports the previous finding by Wessel et al., thereby providing additional evidence for the utility of UWFA. The smaller numbers in our study relative to the one by Wessel et al. [13] might be attributed to the fact that both graders (G. Son and S. Lee) in our study ignored faint peripheral UWFA images thereby excluding possible NP areas.

Among the 101 eyes included in our study, NNV was detected in 10 eyes exclusively outside the 7SF template. Thus, about $10 \%(10 / 101)$ of eyes had NNV lesion that could not be detected using the traditional protocol; it is likely that NV would have been missed in these patients if not for UWFA. By a very rough estimation, we might miss one NNV case in every 10 PDR patients if we only use 7SF.

NP mainly develops in the mid-peripheral retina [16], which lies outside the traditional 7SF protocol. In our study, six eyes also had an NNP area detected entirely outside the 7SF template. Given the inherent feature of this simulated setting, it is expected that UWFA would detects more pathologies than the traditional protocol. Thus, with respect to pathology extent, UWFA detected 2.59 times more NNP area than 7SF. This means that substantial NNP area might have been concealed in the peripheral retina if PDR patients were followed up using only 7SF.

The NP area drives angiogenic factor formation and subsequent fragile new vessel growth [17]. These brittle new vessels can worsen visual prognosis through various potential complications, such as vitreous hemorrhage [17]. For DR treatment of the affected area, we photocoagulate retinal tissue under ischemia to salvage the remaining viable and important areas by suppressing angiogenic factors [18]. In this regard, detecting NP area is clinically critical in terms of screening progression, but it also has therapeutic implications. Moreover, Reddy et al. recently found that UWFA guided-targeted PRP for NV can even prevent new NV [19]. Again, our data supports the utility of UWFA for detecting possible NNP development in the peripheral retina.

DR assumes a chronic clinical course. Therefore, periodic photographs are of utmost importance for understanding disease progression and determining the required treatment. Wessel et al. previously presented the strength of UWFA for elucidating peripheral retinal pathology [13]; however, their trial was a cross-sectional analysis at a single instance. The present study, on the other hand, is the first attempt to determine the usefulness of UWFA for detecting PDR progression using serial check-ups in the same eyes.

It is important to note several inherent limitations of our study. First, selection bias is possible due to its retrospective nature. However, to minimize selection bias, we selected 140 consecutive eyes of 70 patients first by listing all PDR patients who had undergone PRP with UWFA more than three times, without assessing the study images. Furthermore, exclusion criteria were applied to select the 101 final eyes of 67 patients. With obvious confounders, such as medial opacity or artifacts, we only applied a few exclusion criteria after we observed the images. The second limitation is that calculating NNP areas in the peripheral retinal was somewhat arbitrary. Transforming three-dimensional and spherical planes into two-dimensional flat photographs might distort the actual retinal area and the periphery, in particular. The 2.59 ratio of detected NNP area by UWFA compared to 7SF should be reevaluated with this consideration. As the peripheral retina areas are somewhat overestimated due the aforementioned distortion, the actual ratio should be less than 2.59 . However, considering our study design, which naturally allows for more NNP area identification by UWFA, some overestimation with respect to pixel numbers should have minimal impact on our general conclusion. Thirdly, a grader who expected the estimated conclusion might have committed informational bias when drawing the NP area by hand. To minimize this bias, we concealed the purpose of this study to the second grader (S. Lee) until we obtained the data. Moreover, data from this observer was compared to data from the first grader (G. Son), and high inter-grader correlation was obtained ( $>0.8$ Pearson's coefficient). Lastly, 7SF area is simulated template rather actual photographed area and so there could be minor difference between simulated area and actual photography. However, in combining 7 circles we tried to abide by traditional standard field defined in ETDRS as 7 different circles of 30 degree visualization (three horizontally across the macular and four around the optic nerve). And also, those minor differences are not thought to weaken our major point of discussion.

To date, we depend largely on the results from the ETDRS for diagnosing, treating, and following up patients with PDR [17]. The ETDRS is a widely established study, whereas UWFA is a new technology that is only recently being evaluated. A few retrospective studies have identified several possible uses for UWFA in managing DR $[11,13,19]$. However, no current study has analyzed in depth peripheral retinal pathologies found by UWFA. More studies analyzing peripheral retinal pathologies over time, in a quantitative manner, and determining the relationship between UWFA images and clinical course are required. Large scale, prospective randomized control 
Citation: Sohn G, Lee S, Jung HY, Lee KM, Park YS, et al. (2014) Ultra-Wide Field Angiography Improves the Detection of Proliferative Diabetic Retinopathy Progression. J Diabetes Metab 5: 446 doi:10.4172/2155-6156.1000446

Page 5 of 5

studies should be performed to apply our new knowledge from UWFA images to treatment decision making and estimating prognoses.

\section{References}

1. Early photocoagulation for diabetic retinopathy. ETDRS report number 9 . Early Treatment Diabetic Retinopathy Study Research Group. Ophthalmology 98: 766-785.

2. Saaddine JB, Honeycutt AA, Narayan KM, Zhang X, Klein R, et al. (2008) Projection of diabetic retinopathy and other major eye diseases among people with diabetes mellitus: United States, 2005-2050. Arch Ophthalmol 126: 17401747.

3. Classification of diabetic retinopathy from fluorescein angiograms. ETDRS report number 11. Early Treatment Diabetic Retinopathy Study Research Group. Ophthalmology 98: 807-822.

4. Grading diabetic retinopathy from stereoscopic color fundus photographs--an extension of the modified Airlie House classification. ETDRS report number 10. Early Treatment Diabetic Retinopathy Study Research Group. Ophthalmology 98: 786-806.

5. Gangaputra S, Almukhtar T, Glassman AR, Aiello LP, Bressler N, et al. (2011) Comparison of film and digital fundus photographs in eyes of individuals with diabetes mellitus. Invest Ophthalmol Vis Sci 52: 6168-6173

6. Hubbard LD, Sun W, Cleary PA, Danis RP, Hainsworth DP, et al. (2011) Comparison of digital and film grading of diabetic retinopathy severity in the Diabetes Control and Complications Trial/Epidemiology of Diabetes Interventions and Complications Study. Archives of ophthalmology 129: 718726 .

7. Li HK, Danis RP, Hubbard LD, Florez-Arango JF, Esquivel A, et al. (2011) Comparability of digital photography with the ETDRS film protocol for evaluation of diabetic retinopathy severity. Investigative ophthalmology \& visual science 52: 4717-4725

8. Witmer MT, Kiss S (2013) Wide-field imaging of the retina. Surv Ophthalmo 58: $143-154$
9. Neubauer AS, Kernt M, Haritoglou C, Priglinger SG, Kampik A, et al. (2008) Nonmydriatic screening for diabetic retinopathy by ultra-widefield scanning laser ophthalmoscopy (Optomap). Graefes Arch Clin Exp Ophthalmol 246 229-235.

10. Patel M, Kiss S (2014) Ultra-wide-field fluorescein angiography in retinal disease. Curr Opin Ophthalmol 25: 213-220.

11. Friberg TR, Gupta A, Yu J, Huang L, Suner I, et al. (2008) Ultrawide angle fluorescein angiographic imaging: a comparison to conventional digital acquisition systems. Ophthalmic Surg Lasers Imaging 39: 304-311.

12. Oliver SC, Schwartz SD (2010) Peripheral vessel leakage (PVL): a new angiographic finding in diabetic retinopathy identified with ultra wide-field fluorescein angiography. Semin Ophthalmol 25: 27-33.

13. Wessel MM, Aaker GD, Parlitsis G, Cho M, D'Amico DJ, et al. (2012) Ultrawide-field angiography improves the detection and classification of diabetic retinopathy. Retina 32: 785-791.

14. Muqit MM, Marcellino GR, Henson DB, Young LB, Patton N, et al. (2013) Optos-guided pattern scan laser (Pascal)-targeted retinal photocoagulation in proliferative diabetic retinopathy. Acta Ophthalmol 91: 251-258.

15. Control D, Interventions CTEoD, Group CR (2008) Prolonged effect of intensive therapy on the risk of retinopathy complications in patients with type 1 diabetes mellitus: 10 years after the Diabetes Control and Complications Trial. Archives of ophthalmology 126: 1707.

16. Niki T, Muraoka K, Shimizu K (1984) Distribution of capillary nonperfusion in early-stage diabetic retinopathy. Ophthalmology $91: 1431-1439$.

17. Heng LZ, Comyn O, Peto T, Tadros C, Ng E, et al. (2013) Diabetic retinopathy: pathogenesis, clinical grading, management and future developments. Diabet Med 30: 640-650.

18. Abu El-Asrar AM (2013) Evolving strategies in the management of diabetic retinopathy. Middle East Afr J Ophthalmol 20: 273-282.

19. Reddy S, Hu A, Schwartz SD (2009) Ultra Wide Field Fluorescein Angiography Guided Targeted Retinal Photocoagulation (TRP). Semin Ophthalmol 24: 9-14. 